\title{
Observer-Based Robust Control for Spacecraft Rendezvous with Thrust Saturation
}

\author{
Neng Wan, ${ }^{1}$ Ming Liu, ${ }^{1}$ and Hamid Reza Karimi ${ }^{2}$ \\ ${ }^{1}$ Research Center of Satellite Technology, Harbin Institute of Technology, Harbin 150001, China \\ ${ }^{2}$ Department of Engineering, Faculty of Engineering and Science, University of Agder, 4898 Grimstad, Norway \\ Correspondence should be addressed to Ming Liu; robustcontrol23@gmail.com
}

Received 26 February 2014; Accepted 22 April 2014; Published 21 May 2014

Academic Editor: Hongli Dong

Copyright (c) 2014 Neng Wan et al. This is an open access article distributed under the Creative Commons Attribution License, which permits unrestricted use, distribution, and reproduction in any medium, provided the original work is properly cited.

\begin{abstract}
This paper proposes an observer-based robust guaranteed cost control method for thrust-limited rendezvous in near-circular orbits. Treating the noncircularity of the target orbit as a parametric uncertainty, a linearized motion model derived from the two-body problem is adopted as the controlled plant. Based on this model, a robust guaranteed cost observer-controller is synthesized with a less conservative saturation control law, and sufficient condition for the existence of this observer-based rendezvous controller is derived. Finally, an illustrative example with immeasurable velocity states is presented to demonstrate the advantages and effectiveness of the control scheme.
\end{abstract}

\section{Introduction}

As a fundamental operational technology, autonomous rendezvous is widely applied to the astronautic missions involving more than one spacecraft, such as crew exchange, largescale assembly, spacecraft interception, maintenance, docking, and formation flying. Since the autonomous control scheme is a primary factor that determines the success of the rendezvous, it has been and continues to be an attractive area of research.

In order to lower the technical difficulties, routine rendezvous missions are often expected to be conducted in circular orbits. However, an ideal circular orbit is hard to achieve and maintain; as a result, most of the spacecraft rendezvous were actually accomplished in the target orbits with small eccentricities; therefore, adopting a proper and accurate plant model is a prerequisite for the design of the rendezvous controller. The most widely used model for the study of rendezvous was proposed by Clohessy and Wiltshire [1], which is known as the Clohessy-Wiltshire equations (or Hill's equations [2]). Although the form of C-W equations is linear and concise, these equations are only suitable and precise enough for rendezvous in circular orbits. To solve this problem, De Vries [3] and Tschauner [4] proposed the relative motion models for rendezvous in elliptical orbits, which are more accurate than $\mathrm{C}-\mathrm{W}$ equations when reference orbits are noncircular, but nonlinear terms involved in these models would complicate the control task. The concept of rendezvous in near-circular orbits was first raised by Anthony and Sasaki [5], and a linearized modeling method for this kind of rendezvous was investigated by Melton [6]. In consideration of the practical engineering application, a linearized model for rendezvous in near-circular orbits is chosen as the controlled plant in this paper.

In practice, some state variables may not always be available for direct measurement due to physical constraints, such as sensor failure and obstacle blocking. Observer-based control and output feedback control are the two common methods that can cope with this difficulty. Output feedback control method is direct but has many restrictions; more introductions on this method are given in [7-12]. State observers were first proposed and developed by Luenberger [13], since then various kinds of observers including sliding mode observer [14] and fuzzy observer [15] have been developed. Based on these observers, many observer-based control methods have been developed [16-18]. Since state observers 


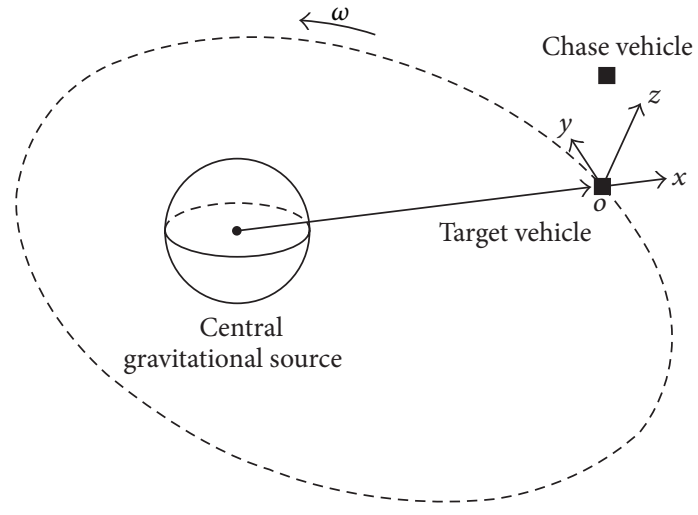

Figure 1: Relative Cartesian coordinate system for spacecraft rendezvous.

have the ability to reconstruct the system states from its output measurements, the observer-based controllers are widely applied to fault diagnosis, fault isolation, reliability improvement, and cost saving by estimating the state variables instead of measuring them by sensors [19-21].

Since the last decades of the 20th century, advanced control theories have been implemented in autonomous rendezvous of spacecraft. Gao et al. [22] designed a multiobjective robust $H_{\infty}$ controller for the spacecraft rendezvous. For the fault-tolerant control problem, Yang and Gao [23] propounded a robust reliable control scheme for thrustlimited rendezvous. Yang and Gao [24] and Sheng et al. [25] synthesized guaranteed cost output tracking controllers for spacecraft rendezvous. Sampled-data controllers for spacecraft rendezvous with thrust nonlinearity were proposed in $[26,27]$. A sampled-data controller designed via a discontinuous Lyapunov's approach was discussed in [28]. By using adaptive output feedback control technique, Singla et al. [29] developed an adaptive control law that is valid for both orbit and attitude control. Bevilacqua et al. [30] provided a control scheme on the basis of Pontryagin's maximum principle; Zhou et al. [31] gave a control method via a parametric Lyapunov's differential equation approach; and a robust tracking controller for relative position holding was studied in our previous work [32]. However, up to now, to the best of the authors' knowledge, an observer-based control scheme for spacecraft rendezvous has not been proposed yet.

To realize spacecraft rendezvous in the situation where not all states are available for feedback, this paper develops an observer-based rendezvous controller. For practicality, a linearized motion model, which is fully suitable for the nearcircular rendezvous but was seldom investigated previously, is used in this paper. A modified saturation control technique, which is more concise but less conservative than those used in $[22,23,25,26,33-38]$, is employed to limit the control forces. Robust guaranteed cost control technique is also utilized to optimize the fuel and time cost in the presence of noncircular uncertainty of the reference orbit. Finally, the observer-based rendezvous controller is cast into a convex optimization problem subject to linear matrix inequality (LMI) constraints, and a numerical example is given to illustrate the usefulness and advantages of our proposed control scheme.

The remainder of this paper is organized as follows. Section 2 sets up the relative motion model and formulates the control problem. Section 3 derives the observerbased controller. Section 4 shows an illustrative example, and Section 5 draws the conclusion.

Notation. The notation used throughout this paper is given below. For a matrix $\mathbf{X}, \mathbf{X}^{T}$ stands for the transpose of $\mathbf{X}$; $\operatorname{sym}(\mathbf{X})$ stands for $\mathbf{X}+\mathbf{X}^{T}$. For a real symmetric matrix $\mathbf{Y}$, the notation $\mathbf{Y}>\mathbf{0}(\mathbf{Y}<\mathbf{0})$ is used to denote its positive- (negative-) definiteness. $\operatorname{diag}(\cdots)$ stands for a blockdiagonal matrix. In symmetric block matrices or complex matrix expressions, we use an asterisk $(*)$ to represent a term that is induced by symmetry. I and $\mathbf{0}$, respectively, denote the identity matrix and zero matrix with compatible dimension. If the dimensions of matrices are not explicitly stated, they are assumed to be compatible for algebraic operation.

\section{Motion Analysis and Problem Formulation}

In this section, based on the two-body problem, a dynamic model for rendezvous in near-circular orbits is established. Furthermore, the autonomous control task is converted into an observer-based stabilization problem, and some requirements on the observer-controller are stated.

2.1. Relative Motion Model. Suppose that two spacecrafts moving in almost identical orbits are very close to each other, and they are only influenced by a central gravitational source. The spacecraft that approaches the other by orbital maneuvering is denoted as the chase vehicle, and the target vehicle denotes the other spacecraft that does not maneuver. It is supposed that the propulsive thrusts of the chase vehicle are independent and continue along each axis.

A relative Cartesian coordinate system shown in Figure 1 is defined to describe the relative motion between the target and the chase vehicles. The origin of the coordinate system is settled at the centroid of the target vehicle. The $x$-axis is aligned with the vector from the Earth's center to the origin of the coordinates system. The $z$-axis is aligned with the angular momentum vector of the target orbit, and the $y$-axis completes an orthogonal right-handed system.

By forming state vector $\mathbf{x}(t)=[x, y, z, \dot{x}, \dot{y}, \dot{z}]^{T}$ that contains the position and velocity states of the chase vehicle and the input vector $\mathbf{u}(t)=\left[f_{x}(t), f_{y}(t), f_{z}(t)\right]^{T}$ where $f_{i}(t)$ for $i=x, y, z$ are the chaser's control forces along each axis, the relative motion model for rendezvous in near-circular orbits can be expressed as

$$
\dot{\mathbf{x}}(t)=(\mathbf{A}+\Delta \mathbf{A}) \mathbf{x}(t)+\mathbf{B u}(t),
$$

where 


$$
\begin{aligned}
& \mathbf{A}=\left[\begin{array}{cccccc}
0 & 0 & 0 & 1 & 0 & 0 \\
0 & 0 & 0 & 0 & 1 & 0 \\
0 & 0 & 0 & 0 & 0 & 1 \\
3 n^{2} & 0 & 0 & 0 & 2 n & 0 \\
0 & 0 & 0 & -2 n & 0 & 0 \\
0 & 0 & -n^{2} & 0 & 0 & 0
\end{array}\right] \\
& \mathbf{B}=\frac{1}{m}\left[\begin{array}{lll}
0 & 0 & 0 \\
0 & 0 & 0 \\
0 & 0 & 0 \\
1 & 0 & 0 \\
0 & 1 & 0 \\
0 & 0 & 1
\end{array}\right] \\
& \Delta \mathbf{A}=\left[\begin{array}{cccccc}
0 & 0 & 0 & 0 & 0 & 0 \\
0 & 0 & 0 & 0 & 0 & 0 \\
0 & 0 & 0 & 0 & 0 & 0 \\
10 e n^{2} \cos M & -2 e n^{2} \sin M & 0 & 0 & 4 e n \cos M & 0 \\
2 e n^{2} \sin M & e n^{2} \cos M & 0 & -4 e n \cos M & 0 & 0 \\
0 & 0 & -3 e n^{2} \cos M & 0 & 0 & 0
\end{array}\right] \text {, }
\end{aligned}
$$

where $n$ is the mean motion of the target vehicle; $m$ is the mass of the chase vehicle; $e$ is the eccentricity of the target orbit; $M=n\left(t-t_{p}\right)$ is the mean anomaly of the target vehicle; and $t_{p}$ is the time of periapsis passage. The norm-bounded matrix $\triangle \mathbf{A}$ that determines the shape of the target orbit is defined as noncircular uncertainty and can be factorized as

$$
\Delta \mathbf{A}=\mathbf{E}_{1} \Lambda \mathrm{E}_{2},
$$

where $\mathbf{E}_{1}$ and $\mathbf{E}_{2}$ are two constant matrices with proper dimensions, and time-variant matrix $\boldsymbol{\Lambda}$ is bounded by $\boldsymbol{\Lambda}^{T} \boldsymbol{\Lambda}<$ I. More detailed descriptions and derivations on model (1) can be obtained from our previous work [32].

The advantages of adopting (1) as the plant model are significant. Compared with the models for circular target orbit, as eccentricity of the target orbit is contained in $\Delta \mathbf{A}$, model (1) is more accurate; while compared with the nonlinear model for elliptical orbits, as model (1) is linearized, it is more designer-friendly. Moreover, as most of the rendezvous missions were accomplished in near-circular orbits, this controlled plant is practical and precise enough to describe the regular spacecraft rendezvous.

2.2. Problem Formulation. In order to assess and optimize the performance of the controller, a quadratic cost function $J$ is defined as

$$
J=\int_{0}^{\infty}\left[\mathbf{x}^{T}(t) \mathbf{Q} \mathbf{x}(t)+\mathbf{u}^{\mathrm{T}}(t) \mathbf{R} \mathbf{u}(t)\right] d t,
$$

where state weighting matrix $\mathbf{Q} \in \mathbb{R}^{6 \times 6}$ is a positive symmetric matrix related to the convergence rate of the relative motion states and the smoothness of the rendezvous trajectory, and control weighting matrix $\mathbf{Q} \in \mathbb{R}^{3 \times 3}$ is a positive symmetric matrix related to the fuel cost of the chase vehicle. The minimum quadratic cost means an optimal compromise among the convergence rate of the motion states, the smoothness of the trajectory, and the consumption of the fuel. Matrices $\mathbf{U}_{i}(i=x, y, z)$ are introduced for the purpose of limiting the control forces. Defining $\mathbf{U}_{x}=[1,0,0]^{T}[1,0,0]$, $\mathbf{U}_{y}=[0,1,0]^{T}[0,1,0]$, and $\mathbf{U}_{z}=[0,0,1]^{T}[0,0,1]$, input constraints along each axis can be expressed as

$$
\left|f_{i}\right|=\left|\mathbf{U}_{i} \mathbf{u}(t)\right| \leq u_{i, \max } \quad(i=x, y, z),
$$

where $u_{i \text { max }}$ is the maximum control force that thrusters can produced along the $i$-axis.

According to the relative motion model (1) and some restrictions presented above, the problems to be studied in this paper are stated below.

Based on plant model (1), design an observer-based robust guaranteed cost controller such that the following requirements are met.

(i) Rendezvous system (1) is asymptotically stable at $\mathbf{x}(t)=\mathbf{0}$, which means that the chase vehicle should encounter the target vehicle in spite of the model uncertainty.

(ii) The quadratic cost function $J$ defined in (4) should be the minimum.

(iii) The propulsive control thrusts along each axis $u_{i}(t)$ should not exceed the maximum values $u_{i \text {, max }}$ assigned in (5).

\section{Robust Observer-Based Control}

In this section, a full-order state observer that can estimate the immeasurable states is proposed, and then a robust observer-based guaranteed cost controller with input saturation for autonomous rendezvous in near-circular orbits is 
designed via an LMI method. At the end of this section, a convex optimization problem for solving the observercontroller is presented.

3.1. Observer Design. As some state variables may not be available for feedback during rendezvous, observer is used to estimate those immeasurable states. Integrating the measurable states into a vector, the output vector $\mathbf{y}(t)$ of the rendezvous system is defined as

$$
\mathbf{y}(t)=\mathbf{C x}(x)
$$

where $\mathbf{C}$ is an output matrix related to the measurability of the state variables. According to (1) and (6), a full-order observer can be described by

$$
\dot{\widehat{\mathbf{x}}}=\mathbf{A} \widehat{\mathbf{x}}+\mathbf{B u}(t)+\mathbf{L}[\mathbf{y}(t)-\mathbf{C} \widehat{\mathbf{x}}(t)],
$$

where $\mathbf{L}$ is the observer gain matrix, and $\widehat{\mathbf{x}}(t)$ is the observed state vector.

The error vector $\mathbf{e}(t)$ denotes the difference between $\mathbf{x}(t)$ and $\widehat{\mathbf{x}}(t)$ or

$$
\mathbf{e}(t)=\mathbf{x}(t)-\widehat{\mathbf{x}}(t)
$$

Substituting (1), (6), and (7) into the derivative of (8), we obtain the dynamic model for the error vector

$$
\dot{\mathbf{e}}(t)=(\mathbf{A}-\mathbf{L C}) \mathbf{e}(t)+\Delta \mathbf{A x}(t) .
$$

In spite of the disturbance caused by uncertain matrix $\Delta \mathbf{A}$, when $(\mathbf{A}, \mathbf{C})$ is completely observable, an observer gain matrix $\mathbf{L}$ that ensures the asymptotic stability of $\mathbf{e}(t)$ can be obtained by choosing a proper set of eigenvalues for matrix $(\mathbf{A}-\mathbf{L C})$.
3.2. Robust Observer-Based Controller Design. Considering the observer-based state feedback control law, the control signal can be generated by

$$
\mathbf{u}(t)=-\mathbf{K} \widehat{\mathbf{x}}(t),
$$

where $\mathbf{K} \in \mathbb{R}^{3 \times 6}$ is the state feedback gain matrix. Substituting (10) into plant model (1), we obtain the closed-loop observerbased system as

$$
\dot{\mathbf{x}}(t)=(\mathbf{A}+\Delta \mathbf{A}-\mathbf{B K}) \mathbf{x}(t)+\mathbf{B K e}(t) .
$$

A lemma needed by subsequent derivation is given here, and its proofs and application can be found in [39].

Lemma 1 (see [39]). Given matrices $\mathbf{G}=\mathbf{G}^{T}, \mathbf{D}$, and $\mathbf{E}$ of appropriate dimensions,

$$
\mathbf{G}+\mathbf{D F E}+\mathbf{E}^{T} \mathbf{F}^{T} \mathbf{D}^{T}<\mathbf{0},
$$

for all $\mathbf{F}$ satisfying $\mathbf{F}^{T} \mathbf{F} \leq \mathbf{I}$, if and only if there exists a scalar $\delta>0$ such that

$$
\mathbf{G}+\delta \mathbf{D D}^{T}+\delta^{-1} \mathbf{E}^{T} \mathbf{E}<\mathbf{0} .
$$

An augmented state vector is defined as $\boldsymbol{\xi}(t)=$ $\left[\begin{array}{ll}\mathbf{x}^{T}(t) & \mathbf{e}^{T}(t)\end{array}\right]^{T}$. Then, the following theorem gives a sufficient condition for the existence of an observer-based robust controller with an upper bound on the performance index (4).

Theorem 2. Consider the closed-loop system (11) with the observer-based state feedback control law in (10). For a given initial augmented state vector $\boldsymbol{\xi}(0)$, if there exist a positive symmetric matrix $\mathbf{X} \in \mathbb{R}^{6 \times 6}$, a matrix $\mathbf{Y} \in \mathbb{R}^{3 \times 6}$, and positive scalars $\varepsilon$ and $\rho$ satisfying

$$
\begin{gathered}
{\left[\begin{array}{ccccccc}
\operatorname{sym}(\mathbf{A X}-\mathbf{B Y})+\varepsilon \mathbf{E}_{1} \mathbf{E}_{1}^{T} & \mathbf{B Y}+\varepsilon \mathbf{E}_{1} \mathbf{E}_{1}^{T} & \mathbf{X} \mathbf{E}_{2}^{T} & \mathbf{0} & \mathbf{Y}^{T} & -\mathbf{Y}^{T} & \mathbf{X} \\
* & \operatorname{sym}[(\mathbf{A}-\mathbf{L C}) \mathbf{X}]+\varepsilon \mathbf{E}_{1} \mathbf{E}_{1}^{T} & \mathbf{0} & \mathbf{0} & -\mathbf{Y}^{T} & \mathbf{Y}^{T} & \mathbf{0} \\
* & * & -\varepsilon \mathbf{I} & \mathbf{0} & \mathbf{0} & \mathbf{0} & \mathbf{0} \\
* & * & * & -\varepsilon \mathbf{I} & \mathbf{0} & \mathbf{0} & \mathbf{0} \\
* & * & * & * & -\mathbf{R}^{-1} & \mathbf{0} & \mathbf{0} \\
* & * & * & * & * & -\mathbf{R}^{-1} & \mathbf{0} \\
* & * & * & * & * & * & -\mathbf{Q}^{-1}
\end{array}\right]<\mathbf{0},} \\
\\
{\left[\begin{array}{cc}
-\rho & \boldsymbol{\xi}^{T}(0) \\
* & -\overline{\mathbf{X}}
\end{array}\right]<0,}
\end{gathered}
$$

where

$$
\overline{\mathbf{X}}=\left[\begin{array}{ll}
\mathbf{X} & \mathbf{0} \\
\mathbf{0} & \mathbf{X}
\end{array}\right]
$$

then there exists a proper observer-controller such that the closed-loop system (11) is asymptotically stable at $\boldsymbol{\xi}(t)=\mathbf{0}$, and the quadratic cost function (4) has an upper bound $\rho$.
Proof. Consider the Lyapunov function

$$
V(t)=\left[\begin{array}{l}
\mathbf{x}(t) \\
\mathbf{e}(t)
\end{array}\right]^{T}\left[\begin{array}{ll}
\mathbf{P} & \mathbf{0} \\
\mathbf{0} & \mathbf{P}
\end{array}\right]\left[\begin{array}{l}
\mathbf{x}(t) \\
\mathbf{e}(t)
\end{array}\right],
$$


where $\mathbf{P}$ is a positive symmetric matrix. Substituting (9) and (11) into the derivative of $V(t)$, we have

$$
\begin{aligned}
\dot{V}(t)= & {\left[\begin{array}{l}
\mathbf{x}(t) \\
\mathbf{e}(t)
\end{array}\right]^{T} } \\
& \times\left[\begin{array}{cc}
\operatorname{sym}[\mathbf{P}(\mathbf{A}+\Delta \mathbf{A}-\mathbf{B K})] & \mathbf{P B K}+\Delta \mathbf{A}^{T} \mathbf{P} \\
* & \operatorname{sym}[\mathbf{P}(\mathbf{A}-\mathbf{L C})]
\end{array}\right] \\
& \times\left[\begin{array}{c}
\mathbf{x}(t) \\
\mathbf{e}(t)
\end{array}\right] .
\end{aligned}
$$

As is well known, the asymptotic stability of (15) can be guaranteed by $\dot{V}(t)<0$. Then, suppose there exist matrices $\mathbf{K}, \mathbf{P}, \mathbf{Q}$, and $\mathbf{R}$ ensuring the following inequalities:

$$
\dot{V}(t)<-\left[\mathbf{x}^{T}(t) \mathbf{Q} \mathbf{x}(t)+\mathbf{u}^{T}(t) \mathbf{R u}(t)\right]<0 .
$$

Integrating (19) from 0 to $\infty$ and noticing that $\boldsymbol{\xi}(t) \rightarrow \mathbf{0}$ as $t \rightarrow \infty$, we obtain

$$
0<J=\int_{0}^{\infty}\left[\mathbf{x}^{T}(t) \mathbf{Q} \mathbf{x}(t)+\mathbf{u}^{T}(t) \mathbf{R u}(t)\right] d t \leq V(0)=J_{\max }
$$

where $V(0)$ also denoted as $J_{\max }$ is an upper bound of the performance index (4). Substituting (8), (10), and (18) into inequalities (19), there is

$$
\left[\begin{array}{cc}
\operatorname{sym}[\mathbf{P}(\mathbf{A}+\Delta \mathbf{A}-\mathbf{B K})]+\mathbf{Q}+\mathbf{K}^{T} \mathbf{R K} & \mathbf{P B K}+\Delta \mathbf{A}^{T} \mathbf{P}-\mathbf{K}^{T} \mathbf{R K} \\
* & \operatorname{sym}[\mathbf{P}(\mathbf{A}-\mathbf{L C})]+\mathbf{K}^{T} \mathbf{R K}
\end{array}\right]<\mathbf{0 .}
$$

Rewriting inequality (21), we have

$$
\boldsymbol{\Psi}+\boldsymbol{\Delta} \boldsymbol{\Phi} \mathbf{E}+\mathbf{E}^{T} \boldsymbol{\Phi}^{T} \Delta^{T}<\mathbf{0}
$$

where

$$
\begin{aligned}
& \Psi=\left[\begin{array}{cc}
\operatorname{sym}[\mathbf{P}(\mathbf{A}-\mathbf{B K})]+\mathbf{Q}+\mathbf{K}^{T} \mathbf{R K} & \mathbf{P B K}-\mathbf{K}^{T} \mathbf{R K} \\
* & \operatorname{sym}[\mathbf{P}(\mathbf{A}-\mathbf{L C})]+\mathbf{K}^{T} \mathbf{R K}
\end{array}\right], \\
& \Delta=\left[\begin{array}{ll}
\mathrm{PE}_{1} & 0 \\
\mathrm{PE}_{1} & 0
\end{array}\right] \text {, } \\
& \Phi=\left[\begin{array}{ll}
\Lambda & 0 \\
0 & 0
\end{array}\right] \text {, } \\
& \mathbf{E}=\left[\begin{array}{cc}
\mathbf{E}_{2} & \mathbf{0} \\
\mathbf{0} & \mathbf{0}
\end{array}\right]
\end{aligned}
$$

By Lemma 1, there exists a positive scalar $\varepsilon$ ensuring (22) by

$$
\boldsymbol{\Psi}+\varepsilon \boldsymbol{\Delta} \boldsymbol{\Delta}^{T}+\varepsilon^{-1} \mathbf{E}^{T} \mathbf{E}<\mathbf{0} .
$$

By the Schur complement, inequality (24) is equivalent to

$$
\left[\begin{array}{ll}
\Gamma_{11} & \Gamma_{12} \\
* & \Gamma_{22}
\end{array}\right]<\mathbf{0}
$$

where

$$
\begin{aligned}
& \boldsymbol{\Gamma}_{11}=\left[\begin{array}{cc}
\operatorname{sym}[\mathbf{P}(\mathbf{A}-\mathbf{B K})]+\varepsilon \mathbf{P E}_{1} \mathbf{E}_{1}^{T} \mathbf{P} & \mathbf{P B K}+\varepsilon \mathbf{P E}_{1} \mathbf{E}_{1}^{T} \mathbf{P} \\
* & \operatorname{sym}[\mathbf{P}(\mathbf{A}-\mathbf{L C})]+\varepsilon \mathbf{P E}_{1} \mathbf{E}_{1}^{T} \mathbf{P}
\end{array}\right], \\
& \boldsymbol{\Gamma}_{12}=\left[\begin{array}{ccccc}
\mathbf{E}_{2}^{T} & \mathbf{0} & \mathbf{K}^{T} & -\mathbf{K}^{T} & \mathbf{I} \\
\mathbf{0} & \mathbf{0} & -\mathbf{K}^{T} & \mathbf{K}^{T} & \mathbf{0}
\end{array}\right] \\
& \boldsymbol{\Gamma}_{22}=\operatorname{diag}\left(-\varepsilon \mathbf{I},-\varepsilon \mathbf{I},-\mathbf{R}^{-1},-\mathbf{R}^{-1},-\mathbf{Q}^{-1}\right) \text {. }
\end{aligned}
$$

By defining $\mathbf{X}=\mathbf{P}^{-1}$ and $\mathbf{Y}=\mathbf{K} \mathbf{P}^{-1}$ and pre- and postmultiplying (24) by $\operatorname{diag}\left(\mathbf{P}^{-1}, \mathbf{P}^{-1}, \mathbf{I}\right)$, where $\mathbf{I}$ is an identity matrix with proper dimension, matrix inequality (25) is then transformed into LMI (14).

To minimize $J_{\max }$, assume that there exists a positive scalar $\rho$, which meets

$$
J_{\max }=V(0)=\boldsymbol{\xi}^{T}(0) \overline{\mathbf{P}} \boldsymbol{\xi}(0) \leq \rho,
$$

where

$$
\overline{\mathbf{P}}=\left[\begin{array}{ll}
\mathbf{P} & \mathbf{0} \\
\mathbf{0} & \mathbf{P}
\end{array}\right]
$$

From (27), we know that $\rho$ is also an upper bound of the quadratic cost function $J$ defined in (4). Then, by the Schur complement, the inequality in (27) can be transformed into

$$
\left[\begin{array}{cc}
\rho & \xi^{T}(0) \\
* & -\overline{\mathbf{P}}^{-1}
\end{array}\right]<\mathbf{0}
$$

By defining $\mathbf{X}=\mathbf{P}^{-1}$, matrix inequality (29) is equivalent to LMI (15). This completes the proof.

According to Theorem 2, an observer-controller with an upper bound $\rho$ on performance cost $J$ is obtained, which meets the requirements (i) and (ii). But more constraints are still needed to limit the control forces. On the basis of Theorem 2, Theorem 3 provides a sufficient condition for the existence of a robust guaranteed cost observer-controller with input saturation.

Theorem 3. Consider closed-loop system (11) with the observer-based state feedback control law in (10). For a given initial augmented state vector $\xi(0)$, if there exist a positive 
symmetric matrix $\mathbf{X} \in \mathbb{R}^{6 \times 6}$, a matrix $\mathbf{Y} \in \mathbb{R}^{3 \times 6}$, positive scalars $\varepsilon$ and $\rho$ satisfying (14), and the following inequalities

$$
\begin{gathered}
{\left[\begin{array}{cc}
-\rho^{-1} & \rho^{-1} \boldsymbol{\xi}^{\mathrm{T}}(0) \\
* & -\mathbf{X}
\end{array}\right]<\mathbf{0},} \\
{\left[\begin{array}{ccc}
-\mathbf{X} & \mathbf{0} & \mathbf{Y}^{T} \mathbf{U}_{i}^{T} \\
* & -\mathbf{X} & -\mathbf{Y}^{T} \mathbf{U}_{i}^{T} \\
* & * & -u_{i, \max }^{2} \rho^{-1} \mathbf{I}
\end{array}\right]<\mathbf{0},}
\end{gathered}
$$

where

$$
\overline{\mathbf{X}}=\left[\begin{array}{ll}
\mathbf{X} & \mathbf{0} \\
\mathbf{0} & \mathbf{X}
\end{array}\right]
$$

then there exists an observer-controller such that closed-loop system (11) is asymptotically stable at $\boldsymbol{\xi}(t)=\mathbf{0}$; quadratic cost function (4) has an upper bound $w>0$, and control inputs along each axis are constrained below the maximum control thrust $u_{i, \max }(i=x, y, z)$ given in (5).

Proof. Pre- and postmultiplying (15) by $\operatorname{diag}\left(\rho^{-1}, \mathbf{I}\right)$, where I is an identity matrix with proper dimensions, we obtain LMI (30). As the upper bound of the cost function $\rho$ is a positive scalar, dividing both sides of (27) by $\rho$, we have

$$
\left[\begin{array}{l}
\mathbf{x}(t) \\
\mathbf{e}(t)
\end{array}\right]^{T}\left[\begin{array}{cc}
\rho^{-1} \mathbf{P} & \mathbf{0} \\
\mathbf{0} & \rho^{-1} \mathbf{P}
\end{array}\right]\left[\begin{array}{l}
\mathbf{x}(t) \\
\mathbf{e}(t)
\end{array}\right]<1
$$

Inequality (33) can always hold, if positive scalar $\rho$ is large enough. Squaring both sides of (5) and dividing both sides of the result by $u_{i, \max }^{2}$, we obtain

$$
\left[\begin{array}{c}
\mathbf{x}(t) \\
\mathbf{e}(t)
\end{array}\right]^{T}\left[\begin{array}{cc}
u_{i, \max }^{-2} \mathbf{K}^{T} \mathbf{U}_{i}^{T} \mathbf{U}_{i} \mathbf{K} & -u_{i, \max }^{-2} \mathbf{K}^{T} \mathbf{U}_{i}^{T} \mathbf{U}_{i} \mathbf{K} \\
* & u_{i, \max }^{-2} \mathbf{K}^{T} \mathbf{U}_{i}^{T} \mathbf{U}_{i} \mathbf{K}
\end{array}\right]\left[\begin{array}{l}
\mathbf{x}(t) \\
\mathbf{e}(t)
\end{array}\right] \leq 1 .
$$

When (34) holds, the control force along the $i$-axis $(i=$ $x, y, z)$ will be restricted within the maximum thrust $u_{i \text { max }}$. Utilizing inequality (33), inequality (34) can be ensured by

$$
u_{i, \max }^{-2}\left[\begin{array}{cc}
\mathbf{K}^{T} \mathbf{U}_{i}^{T} \mathbf{U}_{i} \mathbf{K} & -\mathbf{K}^{T} \mathbf{U}_{i}^{T} \mathbf{U}_{i} \mathbf{K} \\
* & \mathbf{K}^{T} \mathbf{U}_{i}^{T} \mathbf{U}_{i} \mathbf{K}
\end{array}\right]<\rho^{-1}\left[\begin{array}{cc}
\mathbf{P} & \mathbf{0} \\
\mathbf{0} & \mathbf{P}
\end{array}\right] .
$$

By the Schur complement, inequality (35) is equivalent to

$$
\left[\begin{array}{ccc}
-\mathbf{P} & \mathbf{0} & \mathbf{K}^{T} \mathbf{U}_{i}^{T} \\
* & -\mathbf{P} & -\mathbf{K}^{T} \mathbf{U}_{i}^{T} \\
* & * & -u_{i, \max }^{2} \rho^{-1} \mathbf{I}
\end{array}\right]<\mathbf{0} .
$$

By defining $\mathbf{X}=\mathbf{P}^{-1}$ and $\mathbf{Y}=\mathbf{K} \mathbf{P}^{-1}$ and pre- and postmultiplying (36) by $\operatorname{diag}\left(\mathbf{P}^{-1}, \mathbf{P}^{-1}, \mathbf{I}\right)$, we get LMI (31). This completes the proof.
In order to figure out the upper bound of the performance cost function by commercial software, a standard minimization problem is required. As $\rho^{-1}$ is treated as a variable in Theorem 3, another positive scalar $w$, which meets $0<$ $\rho<w$, is introduced to convert Theorem 3 into a standard minimization problem. By the Schur complement, positive scalar $w$ should meet

$$
\left[\begin{array}{cc}
-w & 1 \\
1 & -\rho^{-1}
\end{array}\right]<\mathbf{0}
$$

Then, the robust guaranteed cost observer-controller with input saturation can be determined by solving the following convex optimization problem:

$$
\begin{array}{rl}
\min _{\varepsilon, \rho^{-1}, \mathbf{X}, \mathbf{Y}} & w, \\
\text { s.t. } & (14),(30),(31) \text { and }(37) .
\end{array}
$$

By solving problem (38), we can obtain an optimal solution that consists of $\varepsilon, \rho, w, \mathbf{X}$, and $\mathbf{Y}$. Finally, the observer-based state feedback gain matrix $\mathbf{K}$ can be determined by $\mathbf{K}=\mathbf{Y} \mathbf{X}^{-1}$.

\section{Illustrative Example}

In this section, an example is presented to illustrate the usefulness and advantages of the rendezvous control scheme proposed in this paper.

First of all, some parameters used in this demonstration example are listed. Consider a pair of adjacent spacecrafts. The target vehicle is assumed to be moving on a low earth orbit (LEO) with perigee radius $r_{p}=6728.140 \mathrm{~km}$ and eccentricity $e=0.01$; thus, its mean motion is $n=1.13 \times$ $10^{-3} \mathrm{rad} / \mathrm{s}$. For the chase vehicle, its mass is set to $200 \mathrm{~kg}$, and the maximum control thrusts along the $x$-, $y$-, and $z$ axis are $40 \mathrm{~N}, 40 \mathrm{~N}$, and $20 \mathrm{~N}$. The initial position of the chase vehicle is $3000 \mathrm{~m},-4000 \mathrm{~m}$, and $20 \mathrm{~m}$ from the target vehicle along the $x$-, $y$-, and $z$-axis, and the initial relative velocities along each axis are $-3 \mathrm{~m} / \mathrm{s}, 4 \mathrm{~m} / \mathrm{s}$, and $-0.02 \mathrm{~m} / \mathrm{s}$. Then, the initial state vector of this rendezvous scene is $\mathbf{x}(0)=$ $[3000,-4000,20,-3,4,-0.02]^{T}$. The weighting matrices $\mathbf{Q}$ and $\mathbf{R}$ in (4) are chosen to be identity matrices in this example.

According to the coefficients given above, 24 out of all 64 possible output matrices are completely observable. In this example, the output matrix in (6) is assigned to

$$
\mathbf{C}=\left[\begin{array}{llllll}
1 & 0 & 0 & 0 & 0 & 0 \\
0 & 1 & 0 & 0 & 0 & 0 \\
0 & 0 & 1 & 0 & 0 & 0
\end{array}\right],
$$




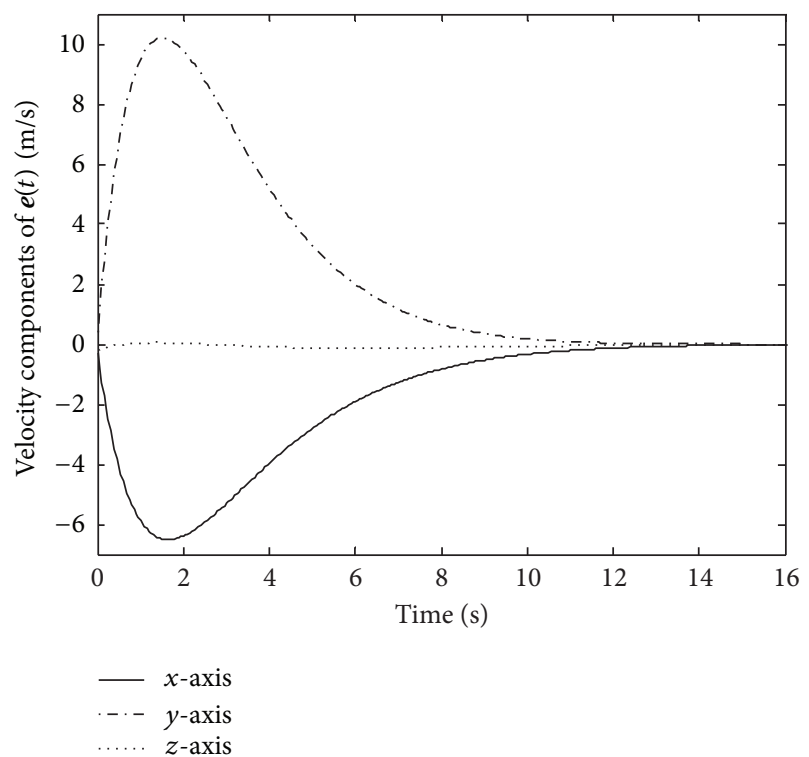

Figure 2: Velocity components of the error vector $\mathbf{e}(t)$.

which implies that the velocity states are not capable of direct measurement. Set the closed-loop poles of the observer to

$$
\begin{gathered}
s_{1,2}=-0.5 \pm j 0.12, \\
s_{3,4}=-0.6 \pm j 0.08, \\
s_{5,6}=-0.75 \pm j 0.1 .
\end{gathered}
$$

By using pole-assignment technique, observer gain matrix $\mathbf{L}$ can be determined as

$$
\mathbf{L}=\left[\begin{array}{ccc}
1.2491 & 0.0127 & 0.0054 \\
-0.025 & 1.2886 & -0.0213 \\
-0.0740 & -0.0388 & 1.1623 \\
0.3863 & 0.0322 & 0.0124 \\
-0.0282 & 0.4142 & -0.0153 \\
-0.0535 & -0.0258 & 0.3440
\end{array}\right]
$$

Assign the matrices in (21) as

$$
\mathbf{E}_{1}=\left[\begin{array}{cccccc}
0 & 0 & 0 & 0 & 0 & 0 \\
0 & 0 & 0 & 0 & 0 & 0 \\
0 & 0 & 0 & 0 & 0 & 0 \\
0 & 2 e & 4 e & 0 & 8 e & 0 \\
2 e & 0 & 0 & 4 e & 0 & 0 \\
0 & 0 & 0 & 0 & 6 e & 0
\end{array}\right],
$$

$$
\mathbf{E}_{2}=\left[\begin{array}{cccccc}
n^{2} & 0 & 0 & 0 & 0 & 0 \\
0 & n^{2} & 0 & 0 & 0 & 0 \\
2.5 n^{2} & 0 & n^{2} & 0 & n & 0 \\
0 & 0.25 n^{2} & 0 & -n & 0 & 0 \\
0 & 0 & n^{2} & 0 & 0 & 0 \\
0 & 0 & 0 & 0 & 0 & n^{2}
\end{array}\right] \text {, }
$$

$\Lambda=\operatorname{diag}(\sin M,-\sin M, \cos M, \cos M,-0.5 \cos M, \cos M)$,

where the mean anomaly $M=n t$. We are now able to compute the state feedback gain matrix $\mathbf{K}$ by solving (38). For brevity, only the crucial parts of the results are listed below

$$
\begin{aligned}
\mathbf{X} & =\left[\begin{array}{cccccc}
0.0079 & -8.8129 \times 10^{-4} & 1.0972 \times 10^{-4} & -3.1524 \times 10^{-5} & 2.0526 \times 10^{-6} & -4.8011 \times 10^{-7} \\
-8.8129 \times 10^{-4} & 0.0079 & 4.0333 \times 10^{-5} & 1.2056 \times 10^{-5} & -3.1936 \times 10^{-5} & 1.4397 \times 10^{-8} \\
1.0972 \times 10^{-4} & 4.0333 \times 10^{-5} & 2.6784 \times 10^{-5} & -3.8559 \times 10^{-7} & -2.5802 \times 10^{-7} & -1.4832 \times 10^{-7} \\
-3.1524 \times 10^{-5} & 1.2056 \times 10^{-5} & -3.8559 \times 10^{-7} & 3.4052 \times 10^{-7} & -1.4787 \times 10^{-7} & 4.5543 \times 10^{-9} \\
2.0526 \times 10^{-6} & -3.1936 \times 10^{-5} & -2.5802 \times 10^{-7} & -1.4787 \times 10^{-7} & 3.0024 \times 10^{-7} & -5.2384 \times 10^{-10} \\
-4.8011 \times 10^{-7} & 1.4397 \times 10^{-8} & -1.4832 \times 10^{-7} & 4.5543 \times 10^{-9} & -5.2384 \times 10^{-10} & 8.4543 \times 10^{-9}
\end{array}\right], \\
\mathbf{Y} & =\left[\begin{array}{cccccc}
2.2713 \times 10^{-5} & -1.9598 \times 10^{-5} & 2.2913 \times 10^{-7} & 3.3538 \times 10^{-8} & -1.6493 \times 10^{-8} & 3.3957 \times 10^{-10} \\
-6.1282 \times 10^{-6} & 3.2079 \times 10^{-6} & -1.4904 \times 10^{-7} & -9.4500 \times 10^{-8} & 1.7293 \times 10^{-7} & -3.1120 \times 10^{-10} \\
-1.7730 \times 10^{-9} & -7.9663 \times 10^{-8} & 6.4364 \times 10^{-7} & 1.0459 \times 10^{-8} & -2.0173 \times 10^{-9} & 1.2532 \times 10^{-8}
\end{array}\right] .
\end{aligned}
$$

Therefore, the observer-based state feedback gain matrix is

$$
\mathbf{K}=\mathbf{Y X}^{-1}=\left[\begin{array}{cccccc}
0.0050 & -0.0035 & 7.2787 \times 10^{-4} & 0.6189 & -0.1539 & 6.8035 \times 10^{-5} \\
-7.7275 \times 10^{-4} & 0.0046 & -1.3303 \times 10^{-4} & -0.0601 & 1.0414 & 0.0060 \\
-2.5577 \times 10^{-4} & -1.5631 \times 10^{-4} & 0.0380 & 0.0429 & 0.0358 & 2.1136
\end{array}\right]
$$

All the simulation results presented below are obtained from a simulation system built on the two-body problem.
As the velocity states are not available for feedback in this example, a full-order state observer (7) is used to estimate 


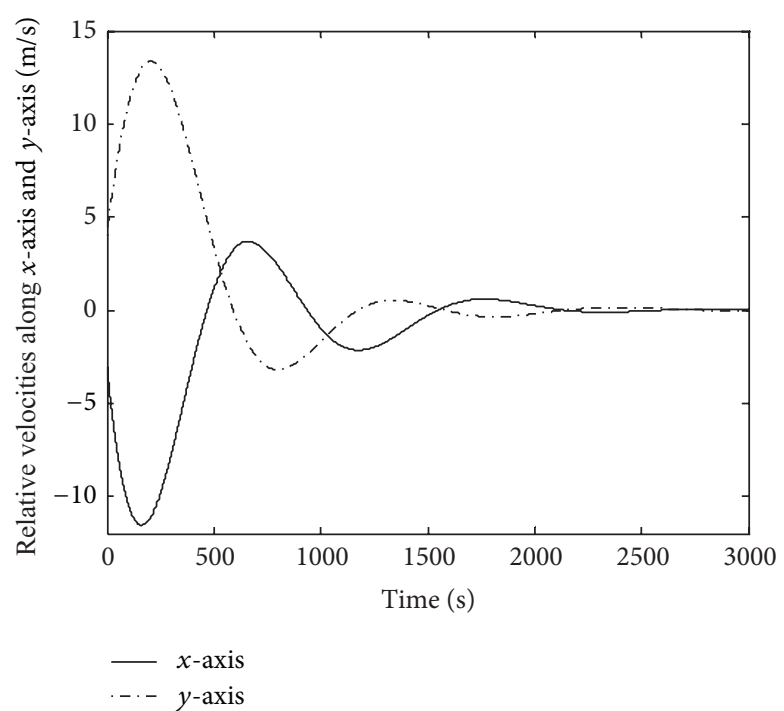

(a)

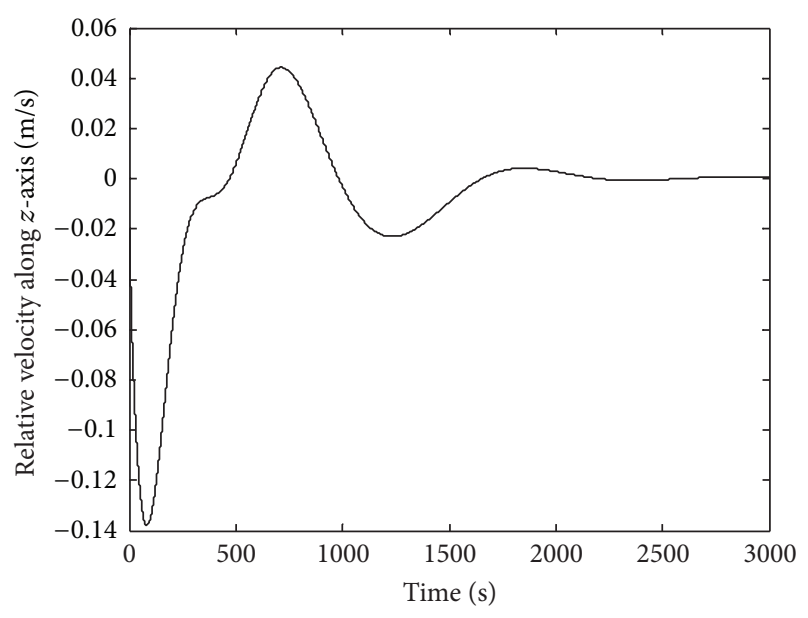

FIGURE 3: Relative velocities between two spacecrafts along each axis.

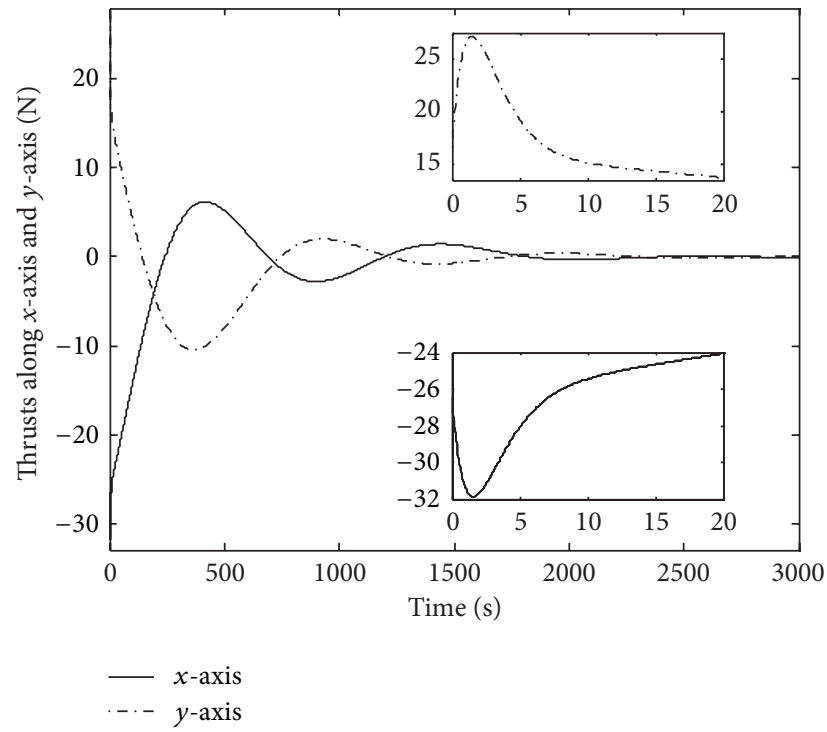

(a)

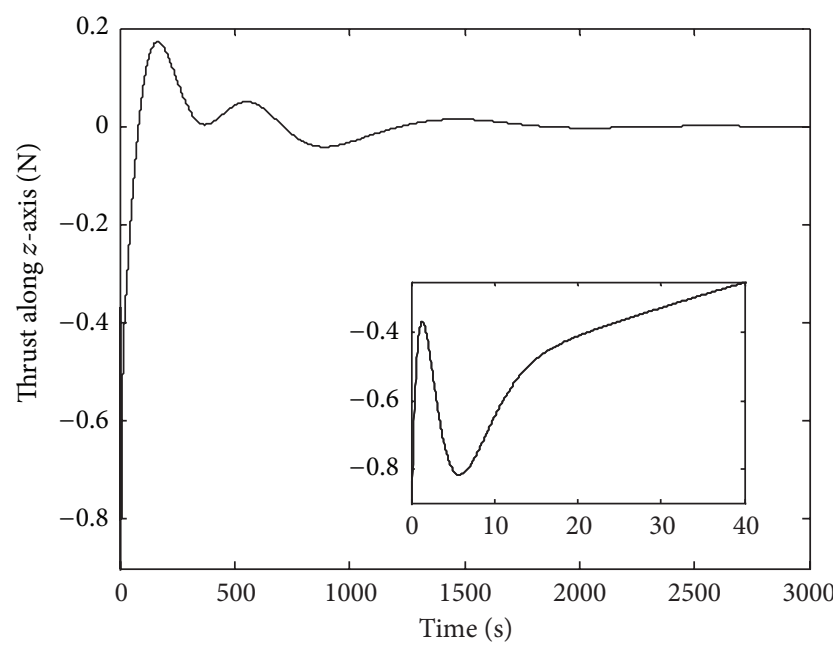

(b)

Figure 4: Propulsive thrusts of the chase vehicle along each axis.

those state variables, and Figure 2 illustrates the errors of the estimated states.

Although the velocity states of the chase vehicle cannot be measured directly, from Figure 2, we can see that the velocity components of the error vector converge to 0 asymptotically and quickly. Compared with the time needed by rendezvous, the convergence time of the error vector is short enough to have little influence on the performance of the whole system.

The velocity states of the chase vehicle during the rendezvous are illustrated in Figure 3. To check if the inputs meet the requirement (iii), Figure 4 shows the control thrusts along each axis, and the rendezvous trajectory of the chase vehicle during the first $3000 \mathrm{~s}$ is depicted in Figure 5.
From Figures 3 and 5, it can be seen that both the measurable and the immeasurable states converge to 0 asymptotically. In Figure 4, the thrusts along each axis are smaller than the bounds given in (5), and the initial fluctuations of the control thrusts are due to the nonzero error vector at the beginning of the rendezvous.

\section{Conclusions}

This paper has discussed a guaranteed cost observer-based control problem for thrust-limited rendezvous in nearcircular orbits. A relative motion model with parametric 


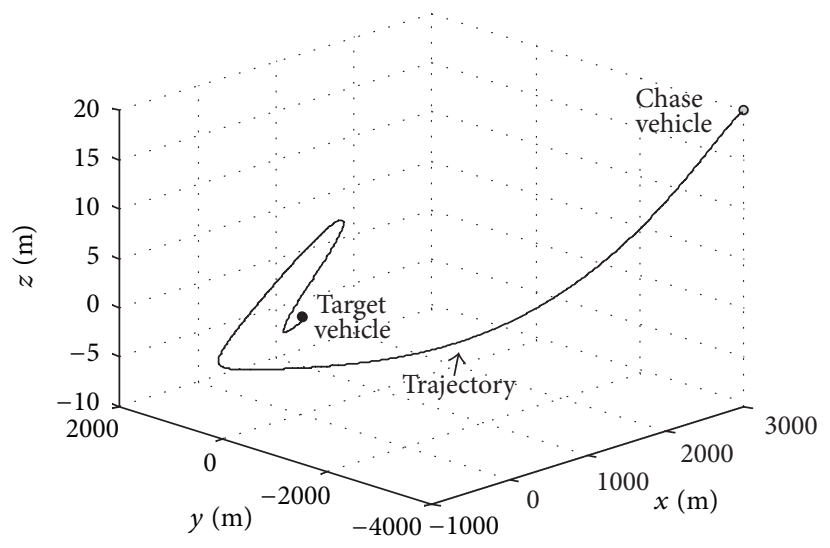

FIGURE 5: Rendezvous trajectory of the chase vehicle.

uncertainty has been used in synthesizing the controller. An optimal observer-based rendezvous controller with a less conservative saturation control law has been developed. An illustrative example has verified the effectiveness and advantages of our proposed control schemes. As it has been shown, this control method can be applied to many routine rendezvous missions for fault isolation and cost saving in the future.

\section{Conflict of Interests}

The authors declare that there is no conflict of interests regarding the publication of this paper.

\section{Acknowledgments}

This work is partially supported by the Norwegian Financial Mechanism 2009-2014 (Grant no. Pol-Nor/200957/47/2013), a grant from Iceland, Liechtenstein, and Norway through the EEA Financial Mechanism operated by Universidad Complutense de Madrid, the National Natural Science Foundation of China (Grant no. 61104101), the China Postdoctoral Science Foundation funded project (Grant no. 2011M500058), the Special Chinese National Postdoctoral Science Foundation (Grant no. 2012T50356), the Heilongjiang Postdoctoral Fund (Grant no. LBH-Z11144), the Open Fund of National Defense Key Discipline Laboratory of Microspacecraft Technology (Grant no. HIT.KLOF.MST.2012006), and Research Fund for the Doctoral Program of Higher Education of China (Grant no. 20112302120011); and the authors wish to thank all the anonymous reviewers and the editor for their valuable comments and suggestions on this paper.

\section{References}

[1] W. H. Clohessy and R. S. Wiltshire, "Terminal guidance system for satellite rendezvous," Journal of the Aerospace Sciences, vol. 31, no. 11, pp. 1516-1521, 1960.

[2] G. W. Hill, "Researches in the Lunar theory," The American Journal of Mathematics, vol. 1, no. 1, pp. 5-26, 1878.
[3] J. P. De Vries, "Elliptic elements in terms of small increments of position and velocity components," AIAA Journal, vol. 1, no. 11, pp. 2626-2629, 1963.

[4] J. Tschauner, "Elliptic orbit rendezvous," AIAA Journal, vol. 5, no. 6, pp. 1110-1113, 1967.

[5] M. L. Anthony and F. T. Sasaki, "Rendezvous problem for nearly circular orbits," AIAA Journal, vol. 3, no. 9, pp. 1666-1673, 1965.

[6] R. G. Melton, "Time-explicit representation of relative motion between elliptical orbits," Journal of Guidance, Control, and Dynamics, vol. 23, no. 4, pp. 604-610, 2000.

[7] C. Scherer, P. Gahinet, and M. Chilali, "Multiobjective outputfeedback control via LMI optimization," Institute of Electrical and Electronics Engineers: Transactions on Automatic Control, vol. 42, no. 7, pp. 896-911, 1997.

[8] D. Zhang, X. Wang, and L. Meng, "Consensus problems for high-order LTI systems: a decentralized static output feedback method," International Journal of Innovative Computing, Information and Control, vol. 9, no. 5, pp. 2143-2154, 2013.

[9] V. Vesely, D. Rosinova, and T. N. Quang, "Networked output feedback robust predictive controller design," International Journal of Innovative Computing, Information and Control, vol. 9, no. 10, pp. 3941-3953, 2013.

[10] L. Wu, X. Su, and P. Shi, "Output feedback control of Markovian jump repeated scalar nonlinear systems," Institute of Electrical and Electronics Engineers: Transactions on Automatic Control, vol. 59, no. 1, pp. 199-204, 2014.

[11] L. Wu, X. Su, and P. Shi, "Sliding mode control with bounded $L_{2}$ gain performance of Markovian jump singular time-delay systems," Automatica, vol. 48, no. 8, pp. 1929-1933, 2012.

[12] X. Su, P. Shi, L. Wu, and Y. Song, "A novel control design on discrete-time Takagi-Sugeno fuzzy systems with time-varying delays," IEEE Transactions on Fuzzy Systems, vol. 21, no. 4, pp. 655-671, 2013.

[13] D. G. Luenberger, "Introduction to observers," IEEE Transactions on Automatic Control, vol. 16, no. 6, pp. 596-602, 1971.

[14] J. J. E. Slotine, J. K. Hedrick, and E. A. Misawa, "On sliding observers for nonlinear systems," in Proceeding of the American Control Conference (IEEE '86), pp. 1794-1800, 1986.

[15] K. Tanaka, T. Ikeda, and H. O. Wang, "Fuzzy regulators and fuzzy observers: relaxed stability conditions and LMI-based designs," IEEE Transactions on Fuzzy Systems, vol. 6, no. 2, pp. 250-265, 1998. 
[16] S. Tong and H.-H. Li, "Observer-based robust fuzzy control of nonlinear systems with parametric uncertainties," Fuzzy Sets and Systems, vol. 131, no. 2, pp. 165-184, 2002.

[17] M. Liu and G. Sun, "Observer-based sliding mode control for Itô stochastic time-delay systems with limited capacity channel," Journal of the Franklin Institute: Engineering and Applied Mathematics, vol. 349, no. 4, pp. 1602-1616, 2012.

[18] M. Liu, P. Shi, L. Zhang, and X. Zhao, "Fault-tolerant control for nonlinear Markovian jump systems via proportional and derivative sliding mode observer technique," IEEE Transactions on Circuits and Systems. I: Regular Papers, vol. 58, no. 11, pp. 2755-2764, 2011.

[19] P. M. Frank and X. Ding, "Survey of robust residual generation and evaluation methods in observer-based fault detection systems," Journal of Process Control, vol. 7, no. 6, pp. 403-424, 1997.

[20] E. A. Garcia and P. M. Frank, "Deterministic nonlinear observer-based approaches to fault diagnosis: a survey," Control Engineering Practice, vol. 5, no. 5, pp. 663-670, 1997.

[21] A. Zhang, J. Ni, and H. R. Karimi, "Reaction wheel installation deviation compensation for overactuated spacecraft with finitetime attitude control," Mathematical Problems in Engineering, vol. 2013, Article ID 268904, 10 pages, 2013.

[22] H. Gao, X. Yang, and P. Shi, "Multi-objective robust Ho control of spacecraft rendezvous," IEEE Transactions on Control Systems Technology, vol. 17, no. 4, pp. 794-802, 2009.

[23] X. Yang and H. Gao, "Robust reliable control for autonomous spacecraft rendezvous with limited-thrust," Aerospace Science and Technology, vol. 24, no. 1, pp. 161-168, 2011.

[24] X. Yang and H. Gao, "Guaranteed cost output tracking control for autonomous homing phase of spacecraft rendezvous," Journal of Aerospace Engineering, vol. 24, no. 4, pp. 478-487, 2011.

[25] D. Sheng, X. Yang, and H. R. Karimi, "Robust control for autonomous spacecraft evacuation with model uncertainty and upper bound of performance with constraints," Mathematical Problems in Engineering, vol. 2014, Article ID 589381, 16 pages, 2014.

[26] X. Yang, X. Cao, and H. Gao, "Sampled-data control for relative position holding of spacecraft rendezvous with thrust nonlinearity," IEEE Transactions on Industrial Electronics, vol. 59, no. 2, pp. 1146-1153, 2012.

[27] Z. Li, M. Liu, H. R. Karimi, and X. Cao, "Observer-based stabilization of spacecraft rendezvous with variable sampling and sensor nonlinearity," Mathematical Problems in Engineering, vol. 2013, Article ID 902452, 11 pages, 2013.

[28] Z. Li, M. Liu, H. R. Karimi, and X. Cao, "Sampled-data control of spacecraft rendezvous with discontinuous Lyapunov approach," Mathematical Problems in Engineering, vol. 2013, Article ID 814271, 10 pages, 2013.

[29] P. Singla, K. Subbarao, and J. L. Junkins, "Adaptive output feedback control for spacecraft rendezvous and docking under measurement uncertainty," Journal of Guidance, Control, and Dynamics, vol. 29, no. 4, pp. 892-902, 2006.

[30] R. Bevilacqua, M. Romano, and O. Yakimenko, "Online generation of quasi-optimal spacecraft rendezvous trajectories," Acta Astronautica, vol. 64, no. 2-3, pp. 345-358, 2009.

[31] B. Zhou, Z. Lin, and G.-R. Duan, "Lyapunov differential equation approach to elliptical orbital rendezvous with constrained controls," Journal of Guidance, Control, and Dynamics, vol. 34, no. 2, pp. 345-358, 2011.
[32] N. Wan, M. Liu, and H. R. Karimi, "Robust tracking control for rendezvous in near-circular orbits," Mathematical Problems in Engineering, vol. 2013, Article ID 726945, 11 pages, 2013.

[33] S. Yin, H. Luo, and S. X. Ding, "Real-time implementation of fault-tolerant control systems with performance optimization," IEEE Transactions on Industrial Electronics, vol. 61, no. 5, pp. 2402-2411, 2014.

[34] S. Yin, S. X. Ding, A. Haghani, H. Hao, and P. Zhang, "A comparison study on basic data-driven fault diagnosis and process monitoring methods on the benchmark Tennessee Eastman process," Journal of Process Control, vol. 22, no. 9, pp. 1567-1581, 2012.

[35] S. Yin, S. X. Ding, A. H. A. Sari, and H. Hao, "Data-driven monitoring for stochastic systems and its application on batch process," International Journal of Systems Science: Principles and Applications of Systems and Integration, vol. 44, no. 7, pp. 13661376, 2013.

[36] H. Dong, Z. Wang, and H. Gao, "Distributed $H_{\infty}$ filtering for a class of Markovian jump nonlinear time-delay systems over lossy sensor networks," IEEE Transactions on Industrial Electronics, vol. 60, no. 10, pp. 4665-4672, 2013.

[37] H. Dong, Z. Wang, and H. Gao, "Distributed filtering for a class of time-varying systems over sensor networks with quantization errors and successive packet dropouts," IEEE Transactions on Signal Processing, vol. 60, no. 6, pp. 3164-3173, 2012.

[38] H. Dong, Z. Wang, and H. Gao, "Fault detection for Markovian jump systems with sensor saturations and randomly varying nonlinearities," IEEE Transactions on Circuits and Systems. I: Regular Papers, vol. 59, no. 10, pp. 2354-2362, 2012.

[39] P. P. Khargonekar, I. R. Petersen, and K. Zhou, "Robust stabilization of uncertain linear systems: quadratic stabilizability and $H_{\infty}$ control theory," Institute of Electrical and Electronics Engineers: Transactions on Automatic Control, vol. 35, no. 3, pp. 356-361, 1990. 


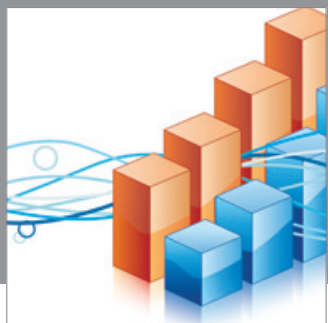

Advances in

Operations Research

mansans

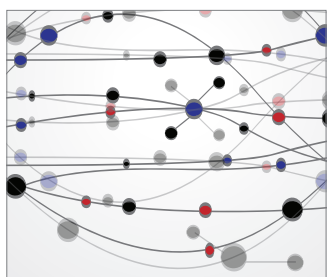

The Scientific World Journal
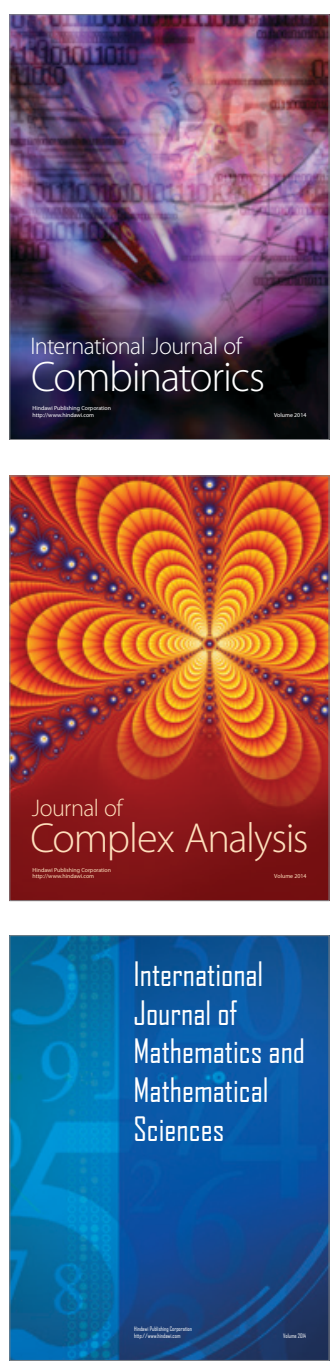
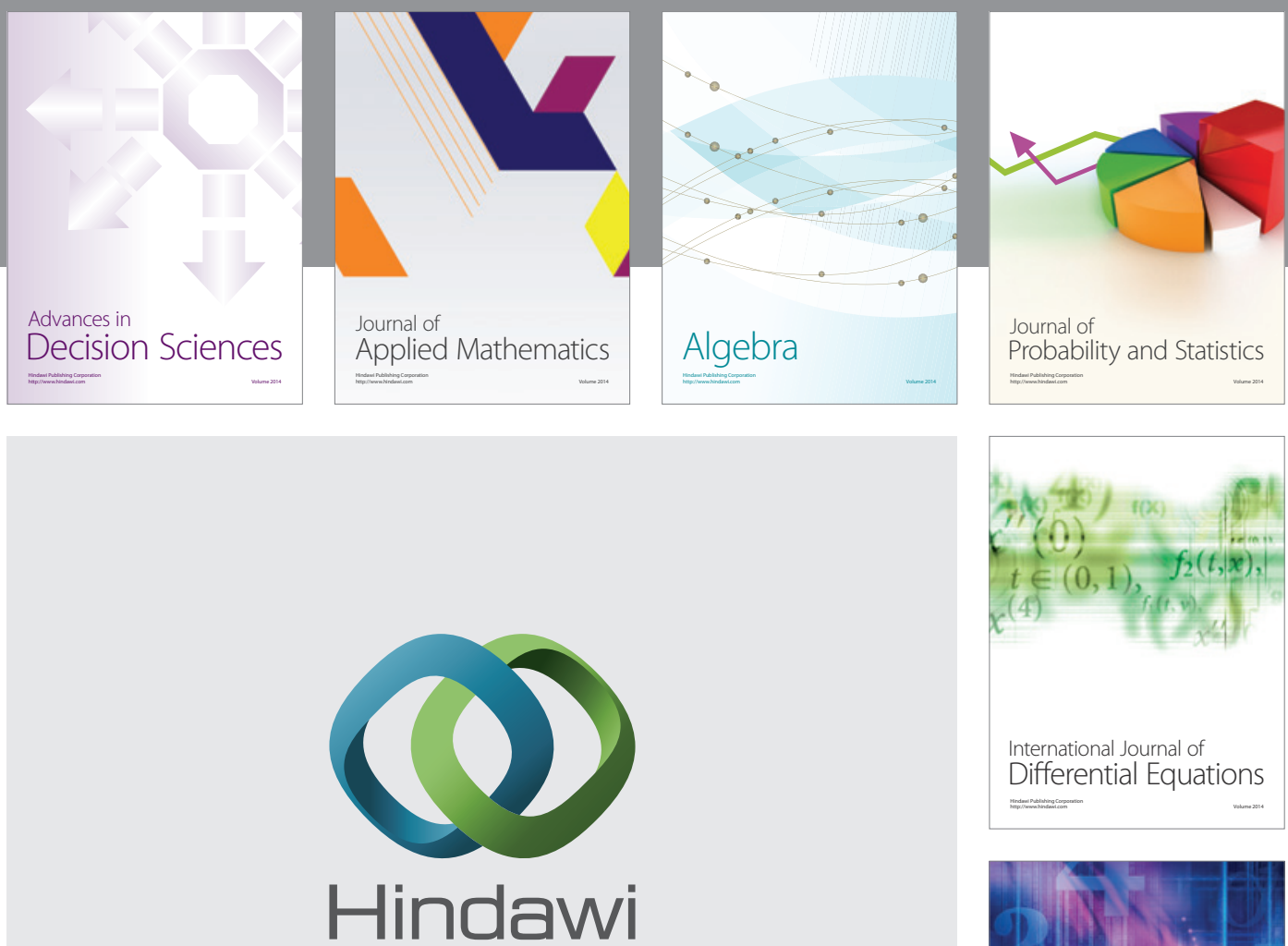

Submit your manuscripts at http://www.hindawi.com
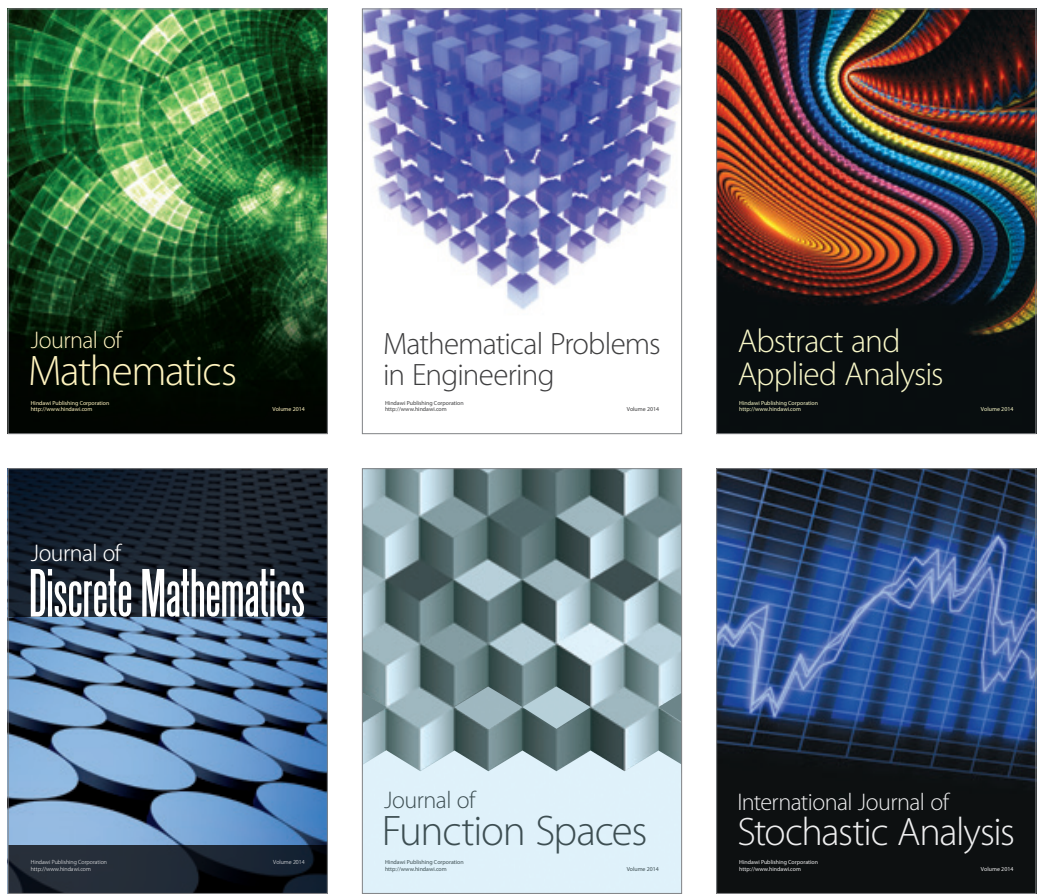

Journal of

Function Spaces

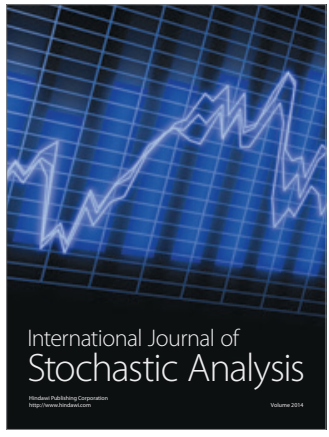

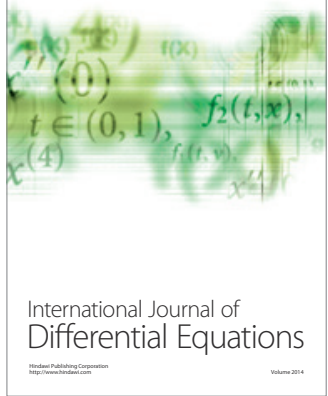
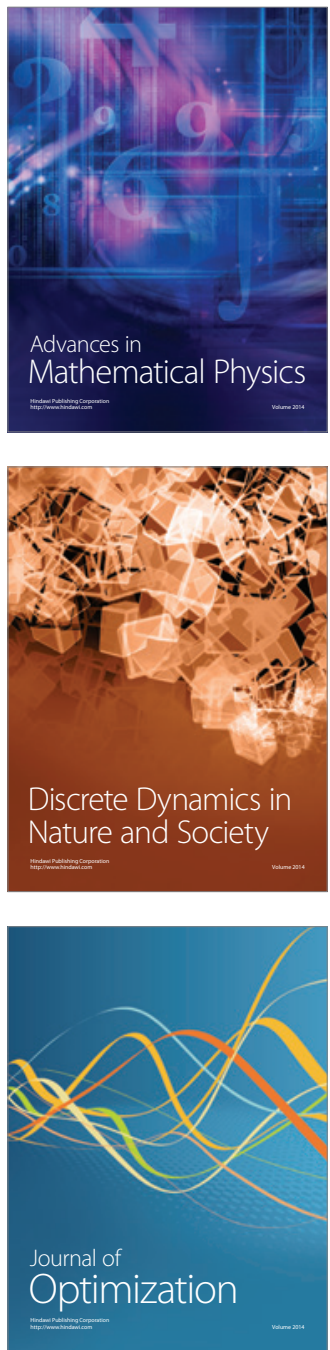